International Game Theory Review

(C) World Scientific Publishing Company

\title{
On the completeness of the universal knowledge-belief space: A technical note
}

\author{
Miklós Pintér \\ Department of Mathematics, Corvinus University of Budapest and \\ MTA-BCE "Lendület" Strategic Interactions Research Group, \\ Fövám tér 13-15, Budapest, 1093, Hungary \\ miklos.pinter@uni-corvinus.hu \\ http://sites.google.com/site/miklospinter/homeeng \\ Received (Day Month Year) \\ Revised (Day Month Year)
}

\begin{abstract}
Meier (2008) shows that the universal knowledge-belief space exists. However, besides the universality there is an other important property might be imposed on knowledgebelief spaces, inherited also from type spaces, the completeness. In this paper we introduce the notion of complete knowledge-belief space, and demonstrate that the universal knowledge-belief space is not complete, that is, some subjective beliefs (probability measures) on the universal knowledge-belief space are not knowledge-belief types.

Keywords: belief; knowledge; uncertainty; complete type space; complete knowledgebelief space; universal type space; universal knowledge-belief space; games with incomplete information.

Subject Classification: C70; C72; D80; D82; D83.
\end{abstract}

\section{Introduction}

To model incomplete information situations Harsányi (1967-68) suggests the use of types instead of hierarchies of beliefs. Summing up his concept very roughly, it is the goal to substitute the belief hierarchies by types, to collect all types in an object, and consider the probability measures on this object as the players' (subjective) beliefs.

In the case of beliefs Heifetz and Samet (1998b) demonstrate the existence of the (purely measurable) universal type space (the object into which all types are collected) and Meier (2012) shows that the universal type space is complete (every probability measure on that is a type).

It is possible to extend type spaces in such a way that they represent not only the beliefs, but the knowledges of the players as well. This extension is called knowledgebelief space.

Meier (2008) introduces the notion of knowledge-belief space, and he succeeds in avoiding the traps of the earlier negative results (Heifetz and Samet (1998a) 
and Brandenburger and Keisler (2006) among others), and shows that the universal knowledge-belief space exists and, naturally, is unique. This important result has been reached by a new, strict, but reasonable definition of the knowledge operator (for the details consult with Meier (2008)).

It is a natural step forward to examine the completeness of the universal knowledge-belief space. For type spaces Brandenburger (2003) introduces the notion of completeness, a type space is complete, if for any subjective belief (probability measure) expressible in the model, there is a type which represents the given subjective belief. The completeness is important because it ensures that we can take any probability measure (subjective belief) and we need not choose of the possible subjective beliefs, which was Harsányi's original goal too.

In this paper we consider Meier (2008)'s universal knowledge-belief space and conclude that is not complete, that is, there are some probability measures (subjective beliefs) on it which cannot be represented by knowledge-belief types.

Our result can be summarized as follows. In the universal knowledge-belief space there is an event (measurable set) which represents $k_{j}\left(k_{i}(\varphi)\right.$ ), that is, player $j$ knows that player $i$ knows that event $\varphi$ happens, and there is another which represents $k_{j}\left(k_{i}(\neg \varphi)\right.$ ), that is, player $j$ knows that player $i$ knows that event $\varphi$ does not happen. However, a probability measure that evaluates these two (disjoint) events equally cannot be in the knowledge-belief space, since $b_{i}^{\frac{1}{2}}\left(k_{j}\left(k_{i}(\varphi)\right)\right)$, that is, player $i$ believes with at least probability of one half that player $j$ knows that player $i$ knows that event $\varphi$ happens, and $b_{i}^{\frac{1}{2}}\left(k_{j}\left(k_{i}(\neg \varphi)\right)\right)$, that is, player $i$ believes with at least probability of one half that player $j$ knows that player $i$ knows that event $\varphi$ does not happen, are not consistent knowledge-belief expressions. Therefore, they cannot be in any state of the world in any knowledge-belief space.

One can see our result in two ways (at least). First, one can interpret our result as the notion of completeness we recommend for the universal knowledge-belief space is futile and not reasonable, since it goes against the very structure of knowledge-belief spaces. However, the notion of completeness we apply to knowledge-belief spaces is not ours, this a simple variant of Brandenburger (2003)'s concept. Therefore, since Brandenburger (2003)'s concept is widely accepted in the literature, perhaps it is not unreasonable to check whether this property holds for the universal knowledgebelief space.

On the other hand, one can say that Meier (2008)'s model is futile and not reasonable, since it does not meet a basic property as completeness. However, it is clear that Meier (2008)'s model has some nice properties (e.g. that there is a universal knowledge-belief space while e.g. there is no universal topological type space (Pintér, 2010)), so perhaps it is not unreasonable to use Meier (2008)'s model either.

We do not want to take sides on this matter just remark the fact that the universal knowledge-belief space is not complete.

We do not introduce every notion used in the paper but refer the reader to 
Meier (2008). Practically, the paper consists of one section, in which we introduce the basic notions and notations and give our non-completeness result in Theorem 2 .

\section{The universal knowledge-belief space is not complete}

Throughout the paper, if not otherwise indicated, we use the terminology and notation of Meier (2008). Moreover we assume that the parameter space $S$ is not trivial, that is, the $\sigma$-field of $S, \Sigma_{S}$, has cardinality more than two, and that there are at least two players.

First we take Definition 2 from Meier (2008).

Definition 1. A knowledge-belief space (kb-space) on $S$ for player set $I$ is a 5 -tuple $\underline{M}:=\left\langle M, \Sigma,\left(K_{i}\right)_{i \in I},\left(T_{i}\right)_{i \in I}, \theta\right\rangle$, where

(1) $M$ is a non-empty set,

(2) $\Sigma$ is a $\sigma$-field on $M$,

(3) $K_{i}: \Sigma \rightarrow \Sigma$ is a knowledge operator ${ }^{\mathrm{a}}$ on $(M, \Sigma)$, for player $i \in I$,

(4) For each $i \in I: T_{i}$ is a $\Sigma$-measurable function from $M$ to $\left(\Delta(M, \Sigma), \Sigma_{\Delta}\right)$, the space of probability measures on $(M, \Sigma)$,

(5) For each $m \in M$ and $E \in \Sigma: m \in K_{i}(E)$ implies $T_{i}(m)(E)=1, i \in I$,

(6) For each $m \in M$ and $E \in \Sigma:\left[T_{i}(m)\right] \subseteq E$ implies $m \in K_{i}(E)$, where $\left[T_{i}(m)\right]:=$ $\left\{m^{\prime} \in M \mid T_{i}\left(m^{\prime}\right)=T_{i}(m)\right\}, i \in I$,

(7) $\theta$ is a $\Sigma$-measurable function from $M$ to $\left(S, \Sigma_{S}\right)$, the parameter space.

If we use the condition "for each $m \in M$ and $E \in \Sigma:\left[T_{i}(m)\right] \subseteq E$ implies $T_{i}(m)(E)=1, i \in I$ " and drop Points (3), (5) and (6) out of Definition 1 then we get the concept of type space (Heifetz and Samet, 1998b), that is, a model where only the beliefs of the players are represented, their knowledge are not.

Definition 2. The knowledge-belief morphism (kb-morphism) $f: M \rightarrow M^{\prime}$ between the $k b$-spaces $\left\langle M, \Sigma,\left(K_{i}\right)_{i \in I},\left(T_{i}\right)_{i \in I}, \theta\right\rangle$ and $\left\langle M^{\prime}, \Sigma^{\prime},\left(K_{i}^{\prime}\right)_{i \in I},\left(T_{i}^{\prime}\right)_{i \in I}, \theta^{\prime}\right\rangle$ is a mapping such that

(1) $f$ is $\Sigma$-measurable,

(2) Diagram (1) is commutative, that is, for all $m \in M: \theta^{\prime} \circ f(m)=\theta(m)$,

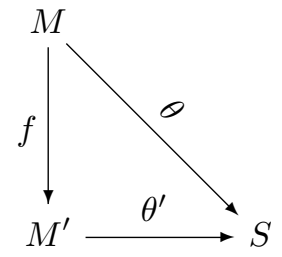

${ }^{a}$ The properties of the knowledge operator is listed in Definition 1. in Meier (2008). 
(3) For each $i \in N$ Diagram (2) is commutative, that is, for all $A \in \Sigma^{\prime}: K_{i} \circ$ $f^{-1}(A)=f^{-1} \circ K_{i}^{\prime}(A)$

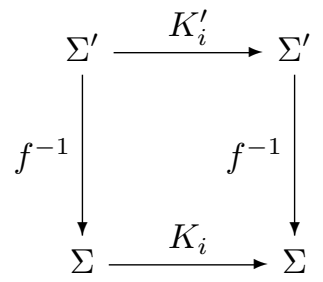

(4) For each $i \in N$ Diagram (3) is commutative, that is, for all $m \in M: T_{i}^{\prime} \circ f(m)=$ $\Delta_{f} \circ T_{i}(m)$,

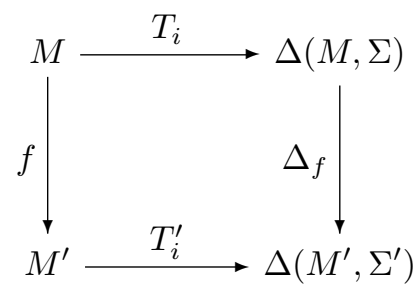

where $\Delta_{f}: \Delta(M, \Sigma) \rightarrow \Delta\left(M^{\prime}, \Sigma^{\prime}\right)$ is defined as for each $\mu \in \Delta(M, \Sigma)$ and $A \in \Sigma^{\prime}$ : $\Delta_{f}(\mu)(A)=\mu\left(f^{-1}(A)\right), i \in N$.

$f$ kb-morphism is a kb-isomorphism, if $f$ is a bijection and $f^{-1}$ is also a $k b$ morphism.

It is easy to verify that ${ }^{\mathrm{b}}$ the kb-spaces as objects and the kb-morphisms as morphisms form a category (the parameter space $\left(S, \Sigma_{S}\right)$ is fixed).

Definition 3. The $k b$-space $\underline{\Omega}$ is universal, if for any $k b$-space $\underline{M}$ there is a unique $k b$-morphism ff from $\underline{M}$ to $\underline{\Omega}$.

In the language of category theory the universal kb-space is a terminal (final) object in the category of kb-spaces. Since every terminal object is unique up to isomorphism, hence the universal kb-space is also unique up to kb-isomorphism.

Theorem 1. The universal kb-space exists.

Proof. See Theorem 1. in Meier (2008).

In other words, Theorem 1 shows that there is a terminal object in the category of kb-spaces.

Definition 4. The kb-space $\underline{M}$ is complete, if for each $i \in I, \mu \in \Delta\left(M, \Sigma_{-i}\right)$ there exists $m \in M$ such that $\mu=\left.T_{i}(m)\right|_{\left(M, \Sigma_{-i}\right)}$, where $\Sigma_{-i}:=\sigma\left(\theta^{-1}\left(\Sigma_{S}\right) \cup\right.$ $\left.\bigcup_{j \in I \backslash\{i\}} K_{j}(\Sigma) \cup \bigcup_{j \in I \backslash\{i\}} T_{j}^{-1}\left(\Sigma_{\Delta}\right)\right)^{\mathrm{c}}$.

${ }^{\mathrm{b}}$ The reason why we use commutative diagrams in Definition 2 is to suggest that category theory is the right framework for analyzing kb-spaces.

${ }^{\mathrm{c}} \sigma(\cdot)$ is for the smallest $\sigma$-field that contains the given set system. 
The above notion of completeness is the natural variant of the one Brandenburger (2003) applies to type spaces.

Intuitively, a kb-space is complete, if for any player any probability measure on the descriptions of the other players and the nature represents a kb-type of the given player. Therefore, if a kb-space is complete, then it is enough to take the probability measures on it; they give correct and full descriptions of the beliefs in the kb-types in the model.

As we have already mentioned in the introduction, the (purely measurable) universal type space is complete (Meier, 2012) and hence it is very desirable that the universal kb-space be also complete. Unfortunately, it does not happen.

Theorem 2. The universal kb-space $\left\langle\Omega, \Sigma_{\Omega},\left(K_{i}^{*}\right)_{i \in I},\left(T_{i}^{*}\right)_{i \in I}, \theta^{*}\right\rangle$ is not complete.

Proof. Let $E \in \Sigma_{S}$ be such that $E, \complement E \neq \emptyset$, and $\varphi \in \Phi$ be the kb-expression that $[\varphi]=\theta^{*-1}(E)^{\mathrm{d}}$. Consider players $i, j \in I, i \neq j$.

Take the following kb-expressions: $k_{j}\left(k_{i}(\varphi)\right)$ and $k_{j}\left(k_{i}(\neg \varphi)\right)$. Then

$$
k_{j}\left(k_{i}(\varphi)\right) \Rightarrow k_{i}(\varphi) \Rightarrow \varphi
$$

and

$$
k_{j}\left(k_{i}(\neg \varphi)\right) \Rightarrow k_{i}(\neg \varphi) \Rightarrow \neg \varphi,
$$

where $\Rightarrow$ is for the implication. Therefore, $\left[k_{j}\left(k_{i}(\varphi)\right)\right],\left[k_{j}\left(k_{i}(\neg \varphi)\right)\right] \in K_{j}\left(\Sigma_{\Omega}\right) \subseteq \Sigma_{-i}$, and $\left[k_{j}\left(k_{i}(\varphi)\right)\right] \cap\left[k_{j}\left(k_{i}(\neg \varphi)\right)\right]=\emptyset$.

Furthermore, $\underline{\Omega}$ is universal kb-space, therefore $k_{i}(\varphi) \Rightarrow k_{i}\left(k_{i}(\varphi)\right)$ (positive introspection) and that $k_{i}(\varphi) \Rightarrow \neg k_{j}\left(k_{i}(\neg \varphi)\right)$ imply that for all $\omega \in \Omega$ :

$$
\begin{array}{r}
k_{i}(\varphi) \in \omega \Rightarrow k_{i}\left(\neg k_{j}\left(k_{i}(\neg \varphi)\right)\right) \in \omega \Rightarrow b_{i}^{1}\left(\neg k_{j}\left(k_{i}(\neg \varphi)\right)\right) \in \omega \\
\Rightarrow \forall p \in(0,1]: \neg b_{i}^{p}\left(k_{j}\left(k_{i}(\neg \varphi)\right)\right) \in \omega .
\end{array}
$$

Analogously, $\neg k_{i}(\varphi) \Rightarrow k_{i}\left(\neg k_{i}(\varphi)\right)$ (negative introspection) and that $\neg k_{i}(\varphi) \Rightarrow$ $\neg k_{j}\left(k_{i}(\varphi)\right)$ imply that for all $\omega \in \Omega$ :

$$
\begin{array}{r}
\neg k_{i}(\varphi) \in \omega \Rightarrow k_{i}\left(\neg k_{j}\left(k_{i}(\varphi)\right)\right) \in \omega \Rightarrow b_{i}^{1}\left(\neg k_{j}\left(k_{i}(\varphi)\right)\right) \in \omega \\
\quad \Rightarrow \forall p \in(0,1]: \neg b_{i}^{p}\left(k_{j}\left(k_{i}(\varphi)\right)\right) \in \omega .
\end{array}
$$

Then it holds for all $\omega \in \Omega\left(\left[k_{i}(\varphi)\right] \cup\left[\neg k_{i}(\varphi)\right]=\Omega\right), p \in(0,1)$ :

$$
b_{i}^{p}\left(k_{j}\left(k_{i}(\varphi)\right)\right) \wedge b_{i}^{1-p}\left(k_{j}\left(k_{i}(\neg \varphi)\right)\right) \notin \omega .
$$

Therefore, if $\mu \in \Delta\left(\Omega, \Sigma_{-i}\right)$ such that $\left(p=\frac{1}{2}\right)$

$$
\left.\mu\left(\left[k_{j}\left(k_{i}(\varphi)\right)\right]\right)=\mu\left(\left[k_{j}\left(k_{i}(\neg \varphi)\right)\right)\right]\right)=\frac{1}{2},
$$

then $\nexists \omega \in \Omega: \mu=\left.T_{i}(\omega)\right|_{\left(M, \Sigma_{-i}\right)}$, that is, the universal kb-space is not complete.

${ }^{\mathrm{d}}$ See Definition 10 in Meier (2008). 
6 REFERENCES

\section{References}

Brandenburger A (2003) On the Existence of a 'Complete' Possibility Structure. In: Basili M, Dimitri N, Gilboa I (eds) Cognitive Processes and Economic Behavior, Routledge, p 30-34

Brandenburger A, Keisler HJ (2006) An impossibility theorem on beliefs in games. Studia Logica 84(2):211-240

Harsányi J (1967-68) Games with incomplete information played by bayesian players part I., II., III. Management Science 14:159-182, 320-334, 486-502

Heifetz A, Samet D (1998a) Knowledge Spaces with Arbitrarily High Rank. Games and Economic Behavior 22(2):260-273

Heifetz A, Samet D (1998b) Topology-free typology of beliefs. Journal of Economic Theory 82:324-341

Meier M (2008) Universal knowledge-belief structures. Games and Economic Behavior 62:53-66

Meier M (2012) An infinitary probability logic for type spaces. Israel Journal of Mathematics 192(1):1-58

Pintér M (2010) The non-existence of a universal topological type space. Journal of Mathematical Economics 46:223-229 